\title{
Trajectory Planning of a Quadrotor to Monitor Dependent People
}

\author{
Lidia M. Belmonte ${ }^{1,2}$, Rafael Morales ${ }^{1,2}$, Arturo S. García ${ }^{1,2}$, Eva Segura ${ }^{1,2}$, \\ Paulo Novais ${ }^{3}$, and Antonio Fernández-Caballero ${ }^{1,2}$ \\ 1 Universidad de Castilla-La Mancha \\ Instituto de Investigación en Informática de Albacete, 02071-Albacete, Spain \\ ${ }^{2}$ Universidad de Castilla-La Mancha \\ Escuela Técnica Superior de Ingenieros Industriales, 02071-Albacete, Spain \\ 3 Universidade do Minho, Escola de Engenharia \\ Campus de Gualtar, 4710-057 Braga, Portugal
}

\begin{abstract}
This article introduces a framework for assisting dependent people at home through a vision-based autonomous unmanned aerial vehicle (UAV). Such an aircraft equipped with onboard cameras can be useful for monitoring and recognizing a dependent's activity. This work is focused on the problem of planning the flight path of a quadrotor to perform monitoring tasks. The objective is to design a trajectory planning algorithm that allows the UAV to position itself for the sake of capturing images of the dependent person's face. These images will be later treated by a base station to evaluate the persons emotional state, together with his/her behavior, this way determining the assistance needed in each situation. Numerical simulations have been carried out to validate the proposed algorithms. The results show the effectiveness of the trajectory planner to generate smooth references to our previously designed GPI (generalized proportional integral) controller. This demonstrates that a quadrotor is able to perform monitoring flights with a high motion precision.
\end{abstract}

Keywords: Home assistance, Dependent people, Unmanned aerial vehicles, Quadrotor, Trajectory planning, Generalized proportional integrated controller.

\section{Introduction}

Inability to perform daily tasks reduces the autonomy and quality of life of dependent people. These people require daily help that has traditionally been provided by health personnel in specialized care centers. However, this kind of care forces dependents to leave their homes, which is an additional problem, since this is not usually the habitual preference. To counteract this situation, family members are usually those who dedicate their time to assist the dependent person. But, in many cases, this is not the ideal solution either. Family caregivers, who cope with a lack of resources and preparation, are sometimes overwhelmed by the situation. Consequently, their quality of life is also affected. 
In addition, every day the number of cases of dependent people living alone is more frequent. Therefore, they must obligatory move to specialized centers to receive the necessary care.

Hence, it is necessary to focus research on the development of home care strategies that allow assistance to dependents. In this way, their personal autonomy is increased. They can stay at home as long as possible and improve their quality of life. In this sense, new technologies provide novel solutions for the care and support of dependents $[11,16,23,25]$. Assistance robotics is fundamental at this point. However, it is essential to work with methods that allow the correct monitoring and identification of the dependent's condition for designing systems that respond to their needs $[28,21]$. One of them is automatic recognition of emotions, a non-invasive method in which our research group has extensive experience $[6,18,13,7]$. This approach requires taking photographs of the person's face for further analyzing the information collected. Thus, the person's mood is detected and the necessary assistance is determined under each situation.

In this context, unmanned aerial vehicles (UAVs) may suppose a new model of home care $[17,30,20]$. Indeed, an UAV equipped with an on-board camera [2, 1], can be very useful in home monitoring. This type of vision-based aircraft allows, unlike other static vision systems, access to remote points, avoid dead angles, and position itself in front of the person [14,15]. The taking of snapshots allows a subsequent recognition of emotions [22]. For this purpose, this article describes a trajectory planner for the flight of a quadrocopter equipped with a camera to capture snapshots of the person's face. The aim is to generate smoothed reference trajectories for a generalized proportional integral (GPI) control algorithm [12], so that the UAV performs the simulation of a flight aimed at monitoring the person. The proposed approach is validated by numerical simulations in MALTAB/Simulink environment [3-5]. This work is part of an ongoing research to design autonomous UAVs for their future use as home assistance for dependent people.

\section{Quadrotor Dynamics}

A quadrotor $[12,31]$ is a rotatory-wing UAV formed by four rotors arranged in the shape of a cross and equidistant from the center of mass of the aircraft, as shown in Fig. 1. Such a vehicle allows vertical take-off and landing, and is characterized by high maneuverability, agility, and versatility. In addition, it can move at low speed, reducing the risk of collision in flight, and improving the quality of the image recorded by a camera aboard. For all these reasons, it has been considered suitable for the proposed approach.

The quadrotor's thrust is generated by the four fixed-angle propellers of the rotors. The lift forces are modified by changing the propellers rotation speed, thus achieving the three possible movements, namely, pitch, roll, and yaw. As shown in Fig. 1, by increasing (reducing) the speed of the propeller [1] while reducing (increasing) the speed of the propeller [3] the pitch movement is obtained. In the case of increasing (reducing) the speed of the propeller [2] while reducing (increasing) the speed of the propeller [4], the roll movement is produced. And 

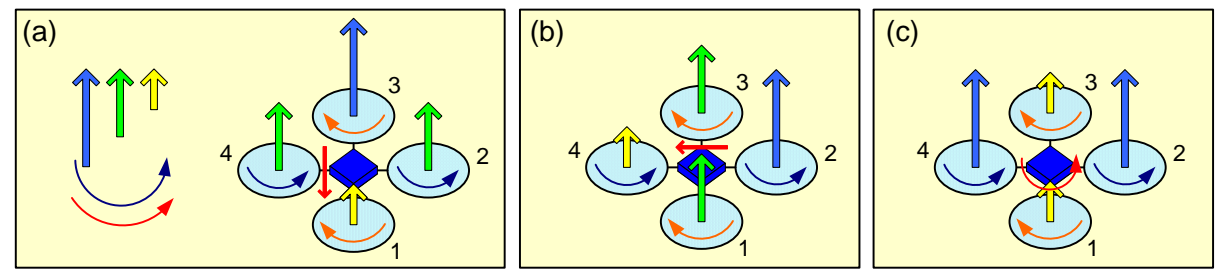

Fig. 1. Quadrotor's motion principles.

finally, by increasing (decreasing) the speed between each pair of propellers, it is possible to modify the yaw angle. The system of equations that model this dynamic behavior has been obtained through the Euler-Lagrange approach [19, 8], resulting in:

$$
\begin{aligned}
& m \ddot{x}=-u \sin \theta \\
& m \ddot{y}=u \cos \theta \sin \phi \\
& m \ddot{z}=u \cos \theta \cos \phi
\end{aligned}
$$$$
\ddot{\psi}=\tau_{\psi}
$$$$
\ddot{\theta}=\tau_{\theta}
$$$$
\ddot{\phi}=\tau_{\phi}
$$

where $m$ is the mass, $g$ is the gravity acceleration, $x$ and $y$ are coordinates in the horizontal plane, $z$ is the vertical position, the angles $\phi, \theta$ and $\psi$ express the independent orientation angles, $u$ is defined as the total thrust and $\tau_{\psi}, \tau_{\theta}$ and $\tau_{\phi}$ denote the angular moments (yawing moment, pitching moment and rolling moment, respectively). Moreover, the following assumption has been considered: orientation angles $\theta$ and $\phi$ are upper and lower bounded in intervals $-\frac{\pi}{2}<\phi<\frac{\pi}{2}$ and $-\frac{\pi}{2}<\theta<\frac{\pi}{2}$.

\section{Control Algorithm}

A control scheme is necessary for regulating and tracking the trajectory that will be generated by the planner (which will be detailed in the next section) to perform a precise flight that allows monitoring a dependent in the proposed assistance system. For this purpose, we have selected a generalized proportional integral (GPI) controller, which is based on the theory of differential flatness, and which has demonstrated good performance in the control of nonlinear systems, which is the case od a quadrotor. GPI control sidesteps the need for traditional asymptotic state observers and proceeds directly to use structural state estimates in place of the actual state variables $[27,26]$. The effect of such structural estimates in the controller is neglected in the feedback control law by means of suitable integral output tracking error feedback control actions.

The complete design of the GPI controller can be consulted in detail in our previous work [12]. The results of this research demonstrated the effectiveness of the proposed approach in comparison with the classical PID control in the following terms: (a) stabilization and trajectory tracking tasks; (b) performance 


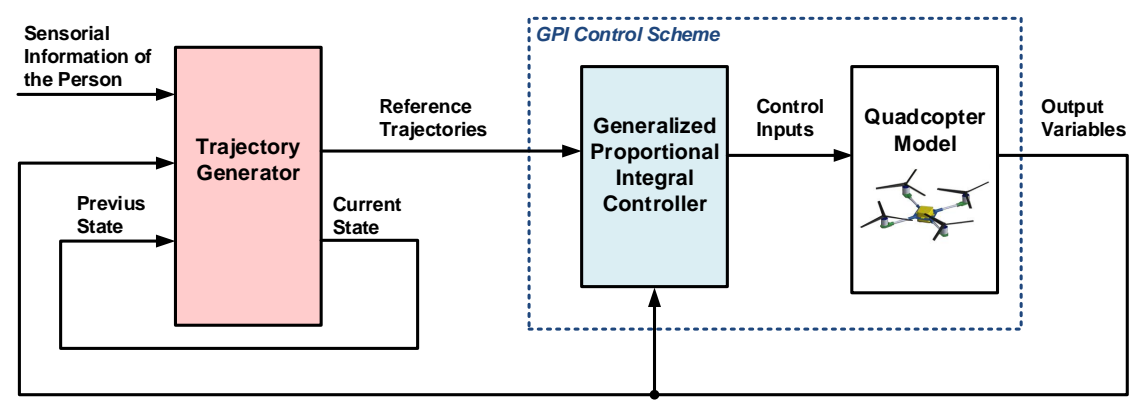

Fig. 2. General control scheme.

when the measured signals are corrupted by noise; and (c) dynamic response when atmospheric disturbances such as gusty wind affect the quadrotor.

\section{Trajectory Planning}

Planning trajectories is one of the problems to necessarily resolve when designing autonomous mechatronic systems and mobile robots. For this reason, it is a field that has attracted the interest of the research community in recent years $[9,10$, 24,29]. Thus, this section describes the trajectory planning algorithm designed for the quadrotor. The overall goal is to make a flight for monitoring a dependent person. To do this, the UAV, which will initially be in a base position on the ground, must take off, approach the person and surround him/her until finding the face. Then, the UAV will take a photograph of the face that will be sent to a base station for analysis. Finally, the UAV must conclude the circular motion around the person and return to its base.

During the planner's development, the following considerations have been considered. (i) The sensors provide the information of the person's position defined by the face's center coordinates $\left(x_{p}, y_{p}, z_{p}\right)$; (ii) the person remains static during the monitoring process; (iii) there are not obstacles at the monitoring height at which the UAV works; (iv) a safety radius, $R$, is defined during the whole monitoring process to avoid collisions between the UAV and the person; and, (v) when the UAV does not perform any monitoring task, it remains in the base position whose coordinates are $\left(x_{b}, y_{b}, z_{b}\right)$.

The trajectory planner is based on a state machine. The states define the maneuvers to be performed by the UAV during the monitoring process. For each state, the planner generates smoothed reference trajectories for the position (coordinates $x, y, z)$ and the yaw angle $(\psi)$ of the UAV. These references are the inputs to the GPI algorithm, which determines the required inputs to control the UAV's flight. The general control scheme of the quadrotor is illustrated in Fig. 2. 
As shown in the figure, the trajectory generator depends on the person's information provided by the sensors $\left(x_{p}, y_{p}, z_{p}\right)$, the UAV's output variables $(x, y, z, \psi)$, and the previous machine state. The planner defines the references trajectories during the monitoring process so that the UAV's camera focus points towards the UAV's forward direction or the person. The considered states for the trajectory planner are the following.

- State 0: Home. It defines the initial state of the UAV located on its base. When it receives the instruction to start the monitoring process, it transits to state 1.

- State 1: Takeoff. Generation of the trajectory for the take-off of the UAV. When the quadrotor reaches the face's height defined by $z_{p}$ coordinate, it transits to state 2 .

- State 2: Person Search. The UAV is requested to rotate its position, that is, to vary its yaw angle to find the person. When the cameras center is aligned with the person, it transits to state 3 .

- State 3: Approximation. The UAV performs an approach maneuver advancing in a straight line towards the person. The objective is to reach the Safety Position located in the circumference of radius $R$ defined around the person. When this position is reached, it transits to state 4 .

- State 4: Waiting in Safety Position. Intermediate state in which the UAV stops before starting the circular movement around the person in order to search his/her face. Once the programmed timeout has elapsed, it transits to state 5 .

- State 5: Face Search. The UAV is requested to perform a circular movement around the person while varying the yaw angle so that the camera on board points towards the person. When the UAV is in front of the face, it transits to state 6 .

- State 6: Data Capture. The UAV stops for a while to take a picture of the person's face. This image is transmitted to a base station for analysis. After the time required for data capture elapses, it transits to state 7 .

- State 7: Motion to Safety Position. Continuation of the circular movement until the turn is completed and the previously defined safety position has been reached. In that position, it transits to state 8 .

- State 8: Base Search. Keeping the position, the UAV is requested to modify its yaw angle until the camera is focused towards the base; then, it transits to state 9 .

- State 9: Return to Base. The UAV must advance in a straight line until it is positioned on the base. When the UAV is on position $\left(x_{b}, y_{b}, z_{p}\right)$, it transits to state 9 .

- State 10: Yaw Angle Adjustment. The UAV is requested to modify its yaw angle so that it can subsequently land on the base correctly and be ready for the next monitoring process.

- State 11: Landing. The UAV lands at the base position and transits to the initial state (0) for the next monitoring process. 


\section{$5 \quad$ Numerical Simulations}

The numerical simulations carried out to evaluate the trajectory planning for the quadrotor are detailed in this section. These simulations were performed within the MATLAB/Simulink environment. The parameters used are defined in Table 1.

Table 1. Parameters defined in the MATLAB/Simulink trials.

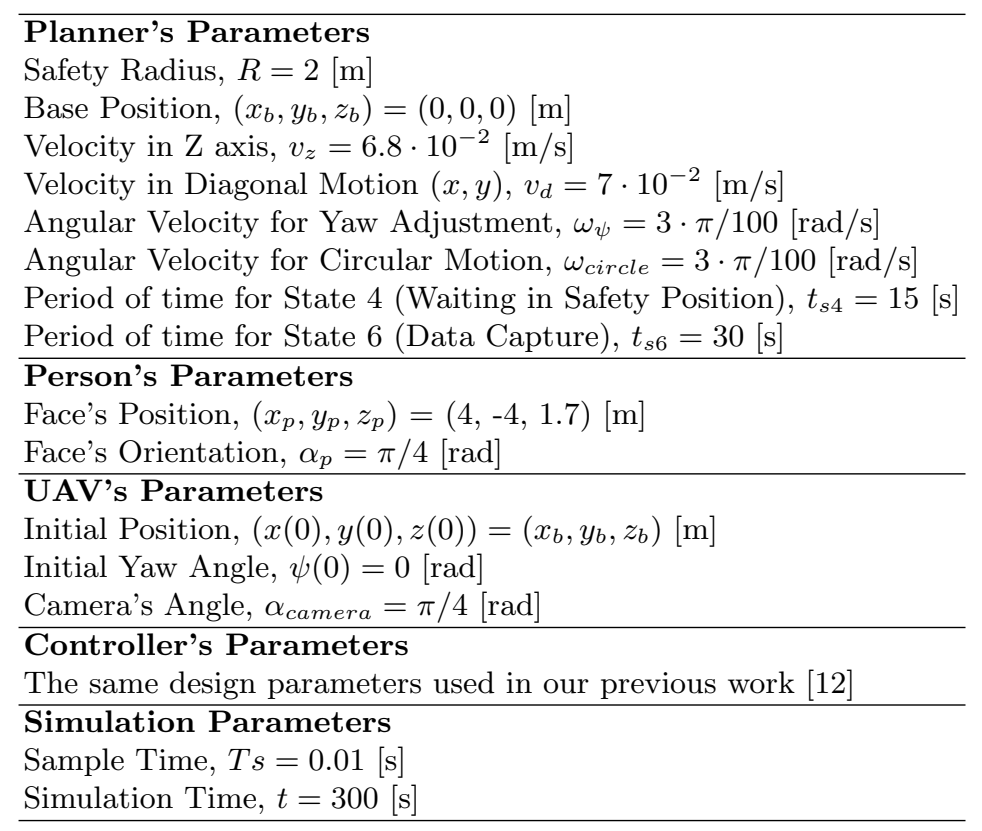

Fig. 3 illustrates the reference trajectory generated by the planner and the trajectory performed by the UAV, both in a $3 \mathrm{D}$ representation. In this picture, the planner's highlight points are detailed. Firstly, there is the base position in which the UAV remains between each monitoring process. Second, we have the person's face position and the direction (where he/she is looking at), which is represented by an arrow. In third place, there is the safety position reached by the UAV in the approximation maneuver and the same position where the UAV returns after taking the photo. Finally, we have the position where the UAV stops to capture that data.

In Fig. 4 and Fig. 5 it is possible to appreciate the precision of the GPI controller for trajectory tracking of the quadrotor's position and orientation. And, finally, the control inputs applied to the UAV model are detailed in Fig. 6. 


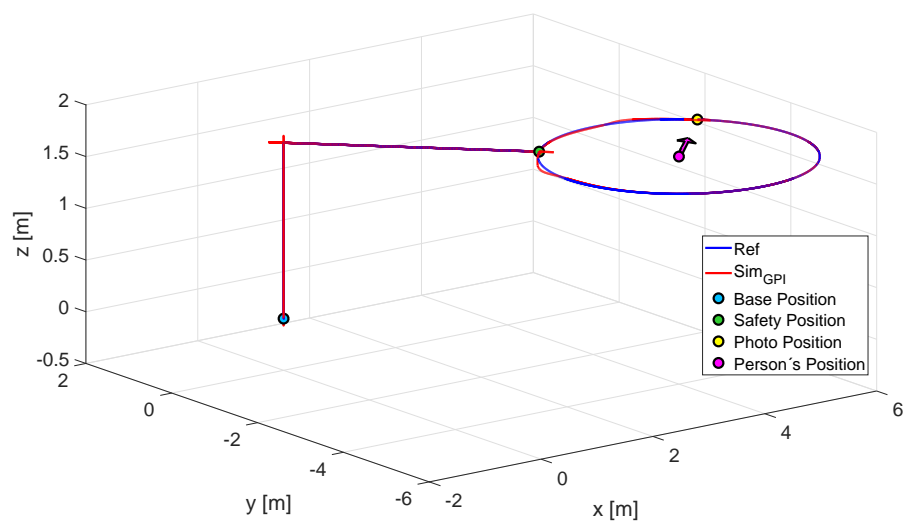

Fig. 3. 3D center of mass of the quadrotor trajectory.
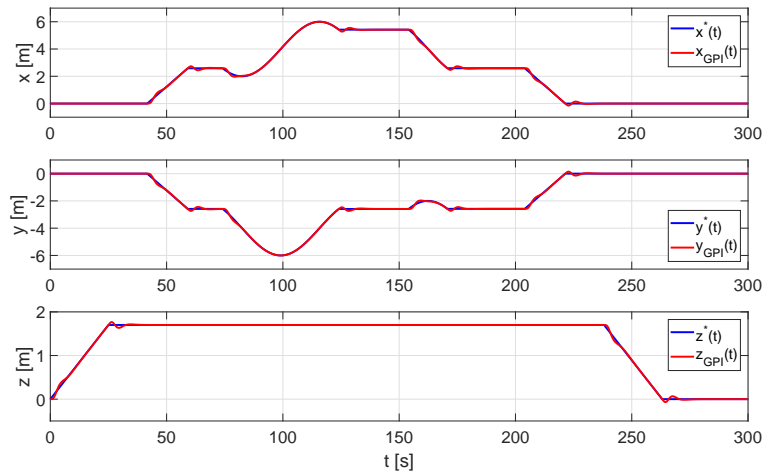

Fig. 4. Position and reference variables of the center of mass of the quadrotor.

\section{Conclusions}

The need of novel home care strategies for dependent people has motivated this work. We have designed a trajectory planner for a quadrotor aimed to monitor dependents. The final aim is to perform an autonomous flight to observe the person and take a photo of his/her face that will be later analyzed to determine the person's mood. That information will allow providing the assistance required at each moment.

Despite being the first development of the trajectory planner, the results of the simulations are positive. The planner is able to generate smoothed reference trajectories that allow performing precise flights of the UAV governed by a GPI controller. In future works, it will be necessary to improve the planner in the following aspects: (a) to increase the planner's detail to consider the tran- 

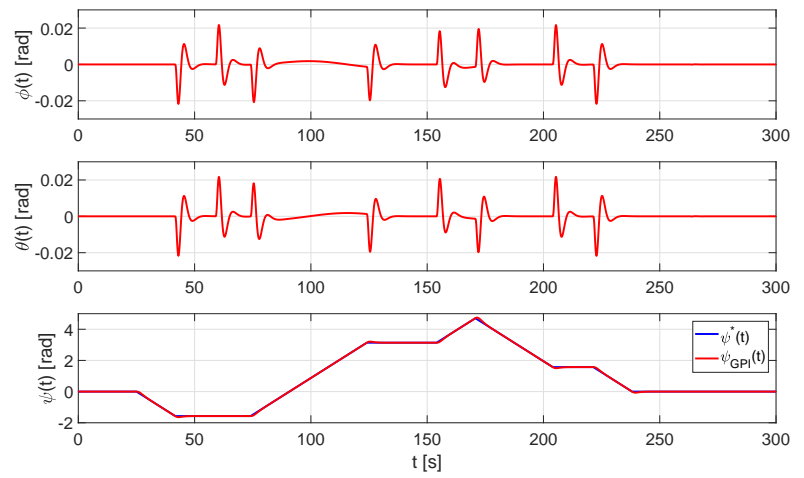

Fig. 5. Attitude variables of the quadrotor.

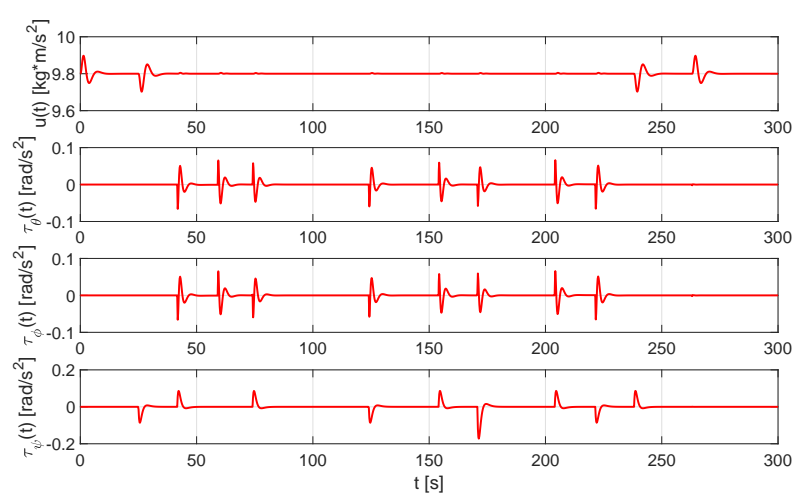

Fig. 6. Applied control inputs.

sitions between states as a consequence of the movement of the person during the monitoring process; (b) to develop a strategy for detecting obstacles and avoiding collisions in the flight environment; (c) to perform an experimentation of the proposed approach in virtual reality environments before moving to real scenarios.

\section{Acknowledgments}

This work has been partially supported by Spanish Ministerio de Ciencia, Innovación y Universidades, Agencia Estatal de Investigación (AEI) / European Regional Development Fund (FEDER, UE) under DPI2016-80894-R grant. Lidia M. Belmonte holds FPU014/05283 scholarship from Spanish Ministerio de Educación y Formación Profesional. 


\section{References}

1. S. Almansa-Valverde, J.C. Castillo, A. Fernndez-Caballero, "Mobile robot map building from time-of-flight camera," Expert Syst. Appl. 39(10), 8835-8843, 2012.

2. L.M. Belmonte, J.C. Castillo, A. Fernández-Caballero, S. Almansa-Valverde, R. Morales, "Flying depth camera for indoor mapping and localization," Ambient Intelligence - Software and Applications, 376, 243-251. Springer, 2015.

3. L.M. Belmonte, R. Morales, A. Fernández-Caballero, J.A. Somolinos, "A tandem active disturbance rejection control for a laboratory helicopter with variable speed rotors," IEEE Trans. Ind. Electron. 63(10), 6395-6406, 2016.

4. L.M. Belmonte, R. Morales, A. Fernández-Caballero, J.A. Somolinos, "Robust decentralized nonlinear control for a twin rotor MIMO system," Sensors 16(8), article 1160, 2016.

5. L.M. Belmonte, R. Morales, A. Fernández-Caballero, J.A. Somolinos. "Nonlinear cascade-based control for a twin rotor MIMO system," Nonlinear Systems - Design, Analysis, Estimation and Control, 265-292. In-Tech, 2016.

6. J.C. Castillo, Á. Castro-González, F. Alonso-Martín, A. Fernández-Caballero, M.A. Salichs, "Emotion detection and regulation from personal assistant robot in smart environment," in Personal Assistants: Emerging Computational Technologies, 179-195. Springer, 2018.

7. J.C. Castillo, Á. Castro-González, A. Fernández-Caballero, J.M. Latorre, J.M. Pastor, A. Fernández-Sotos, M.A. Salichs, "Software architecture for smart emotion recognition and regulation of the ageing adult," Cogn. Comput. 8(2), 357-367, 2016.

8. P. Castillo, A. Dzul and R. Lozano. "Real-time stabilization and tracking of a four rotor mini rotorcraft," IEEE Trans. Control Syst. 12(4), 510-516, 2004.

9. J. Chocoteco, R. Morales, V. Feliu, "Enhancing the trajectory generation of a stair-climbing mobility system," Sensors 17(1), 1-31, 2017.

10. J. Chocoteco, R. Morales, V. Feliu and L. Sánchez, "Trajectory planning for a stair-climbing mobility system using laser distance sensors," IEEE Sens. J. 10(3), 944-956, 2016.

11. J. Chocoteco, R. Morales, V. Feliu, "Improving the climbing/descent performance of stair-climbing systems confronting architectural barriers with geometric disturbances," Mechatronics 30, 11-26, 2015.

12. A. Fernández-Caballero, L.M. Belmonte, R. Morales, J.A. Somolinos, "Generalized proportional integral control for an unmanned quadrotor system," Int. J. Adv. Robot. Syst. 12, article 85, 2015.

13. A. Fernández-Caballero, A. Martínez-Rodrigo, J.M. Pastor, J. C. Castillo, E. Lozano-Monasor, M. T. López, R. Zangróniz, J. M. Latorre, A. Fernández-Sotos, "Smart environment architecture for emotion recognition and regulation," J. Biomed. Inform., 64, 55-73, 2016.

14. J.M. Gascueña, A. Fernández-Caballero, "Agent-oriented modeling and development of a person-following mobile robot," Expert Syst. Appl. 38(4), 4280-4290, 2011.

15. J.M. Gascueña, A. Fernández-Caballero, "Agent-based modeling of a mobile robot to detect and follow humans," 4th International KES Symposium on Agents and Multi-agent Systems Technologies and Applications, 80-89, Springer, 2009.

16. R. Igual, I. Plaza, C. Medrano, M. A. Rubio, "Personalizable smartphone-based system adapted to assist dependent people," J. Ambient. Intell. Smart Environ. 6(6), 569-593, 2014. 
17. S.J. Kim, G. J. Lim, J. Cho, M. J. Côté, "Drone-aided healthcare services for patients with chronic diseases in rural areas," J. Intell. Robot. Syst, 88(1), 163$180,2017$.

18. E. Lozano-Monasor, M.T. López, F. Vigo-Bustos, A. Fernández-Caballero, "Facial expression recognition in ageing adults: From lab to ambient assisted living," J. Ambient. Intell. Humaniz. Comput. 8(4), 567-578, 2017.

19. R. Lozano. "Unmanned Aerial Vehicles-Embedded Control," John Wiley, 2010.

20. C. Todd, A. Lutfi, A. Copiaco, V. Agarwal, K. Afsari, C. Johnathon, O. Okafor, M. Ayad, "Towards an autonomous UAV for indoor flight in healthcare: A review of research challenges and approaches," Int. J. Emerging Technol. Adv. Eng. 5(8), 21-28, 2015

21. I. Maglogiannis, C. Ioannou, P. Tsanakas, "Fall detection and activity identification using wearable and hand-held devices," Integr. Comput. Aided Eng. 23(2), 161-172, 2016

22. J. Martínez-Gómez, A. Fernández-Caballero, I. García-Varea, L. Rodríguez, C. Romero-González, "A taxonomy of vision systems for ground mobile robots," Int. J. Adv. Robot. Syst. 11, 111, 2014.

23. R. Morales, J. Chocoteco, V. Feliu, H. Sira-Ramrez. "Obstacle surpassing and posture control of a stair-climbing robotic mechanism," Control Eng. Pract. 21, 604-621, 2013.

24. R. Morales, V. Feliu, A. González. "Optimized Obstacle avoidance trajectory generation for a reconfigurable staircase climbing wheelchair," Robot. Auton. Syst. 58, 97-114, 2010.

25. R. Morales, A. González, V. Feliu, P. Pintado, "Environment adaptation of a new staircase climbing eheelchair," Auton. Robot. 23, 275-292, 2007.

26. R. Morales, H. Sira-Ramírez. "Trajectory tracking for the magnetic ball levitation system via exact feedforward linearization and GPI control," Int. J. Control 83, 1155-1166, 2010.

27. R. Morales, H. Sira-Ramírez , V. Feliu, A. González. "Adaptive control based on fast online algebraic identification and GPI control for magnetic levitation systems with time-varying input gain," Int. J. Control 87, 1604-1621, 2014.

28. F. J. Rodriguez, F. M. Rico, V. M. Olivera, "Neural networks for recognizing human activities in home-like environments," Integr. Comput. Aided Eng. 26(1), 37-47, 2019

29. R. Solea, U. Nunes, "Trajectory planning and sliding-mode control based trajectory-tracking for cybercars," Integr. Comput. Aided Eng. 14(1), 33-47, 2007.

30. C. Todd, M. Watfa, Y. El Mouden, S. Sahir, A. Ali, A. Niavarani, A. Lutfi, A. Copiaco, V. Agarwal, K. Afsari, C. Johnathon, O. Okafor, M. Ayad, "A proposed UAV for indoor patient care," Technol. Health Care, doi:10.3233/THC1046, 2015.

31. K. M. Zemalache, L. Lotfi H. Maaref, Hichem, "Two inertial models of X4-flyers dynamics, motion planning and control," Integr. Comput. Aided Eng. 14(2), 107119, 2007 . 